\title{
GESMO- MOtion GESture based Mobile Application
}

\author{
Ravindra J. Mandale \\ Assistant Professor \\ RIT, Rajaramnagar \\ Maharashtra, India
}

\author{
Sagar Pawar \\ Software Engineer \\ Persistent Systems Limited \\ Maharashtra, India
}

\author{
Vikram Chavan \\ RIT, Rajaramnagar \\ Islampur, Maharashtra, \\ India
}

\begin{abstract}
There has been number of android mobile apps available for clients to solve their daily problems. This paper presents a Motion Gesture based mobile (GESMO) app in Android which allows user to draw gestures in the air resulting opening of a desired app assigned to corresponding gesture. This gesture may be the first letter of an app name which is to be launched. In order to start the motion, user must press and hold any point on the screen until the motion is completed. Once motion completes, it is released. For sensing the motion, we used two built-in sensors from mobile devices: accelerometer and gyroscope. The paper proposes a two-stage approach for spotting and recognition of generated stroke gesture. The spotting stage uses a Support Vector Machine (SVM) to identify data fragments containing one stroke gesture. The recognition stage uses Hidden Markov Models (HMM) to generate the text representation from the motion sensor data. With this technique, you can successfully receive $70-80 \%$ accuracy in detecting an air gesture for mobile devices.
\end{abstract}

\section{General Terms}

Mobile Application Development, Pattern Recognition et. al.

\section{Keywords}

Motion Sensors, Air Gestures, Support Vector Machine (SVM), Hidden Markov Models (HMM).

\section{INTRODUCTION}

Mobile computing is the most interesting and enterprising topic in the current era of research and innovation. Mobile computing is defined as a computation on mobile devices such as mobile phones, tablets and smart phones. In present times, everyone is addicted in using mobile devices; however they are useful in our daily life to accomplish needful tasks by sitting at home on finger touch. Almost from child to adult people rely on these devices to complete their work. These mobile devices require operating system to run their applications. There are number of mobile operating systems available like Google's Android, Nokia's Symbian, Apple's IOS, Microsoft's Windows Mobile, Palm OS, Samsung's Bada, etc. But Google's Android is the most popular operating system for mobile devices all over the world [1]. In actual, Android was developed by Andy Rubin in 2003 at a small start-up company named Android Inc. In July 2005, Google purchased Android and in collaboration with Open Handset Alliance (OHA) released their first official version in 2007 called Cupcake. This OHA consists of more than 80 companies including Motorola, Sony, etc. and it is led by Google Inc. Android comprises not only operating system but also middleware and key applications. The latest release of Android operating system is Marshmallow 6.0

\section{RELATED WORK}

Gesture recognition in the air is very famous and innovative area of research in the field of computer science and language technology; also it added many applications in ubiquitous computing environment.

Ankita Saxena et al. presented [2] technique for gesture recognition which uses artificial neural network among back propagation algorithm. This technique consists of five phases: image acquisition, image preprocessing i.e. image detection, thining, hand token, training data and recognition. They used Sobel detector for detecting edges of an images.

Jiahui $\mathrm{Wu}$ et al. proposed [3] an acceleration based gesture recognition approach called Frame Based Descriptor and multi-SVM (FDSVM) which needs only a wearable 3-D accelerometer as a requirement. This system has four main components: acceleration data acquisition, feature extraction, training phase, and recognition phase. This paper concluded stating SVM gives better results than other four methods including HMM in user- independent and user-dependent cases.

Sandip Agrawal et al. proposed [4] technique to mimic a pen called PhonePoint Pen for giving input to mobile devices. They tested this technique through Nokia 95 Model and detected the alphabets created in the air. They defined new metric called Character Recognition Accuracy (CRA) and tested this accuracy for model.

Po-Kuan Huang et al. proposed [5] stereo matching model which optimized for $3 \mathrm{D}$ hand gesture recognition system. They implemented gesture mouse application which accepts depth map video stream. This enables user to give finger clicks and use regular mouse functions.

Christoph Amma et al. presented [6] new input method for mobile device by creating air gesture with the use of inertial sensors at the back of hand. They used HMM in recognition phase to generate the text representation from the motion sensor data. A statistical language model is used to enhance recognition performance and restrict the search space.

Meenaakumari and Muthulakshmi presented [7] Micro Electro Mechanical Systems (MEMS) accelerometer for character gesturing sensing and sent information to computer for trajectory algorithm. This algorithm consists of information assortment algorithm, signal preprocessing. etc. After completing the above survey, we decided to provide innovative input methodology to mobile devices.

\section{MOTION GESTURE BASED MOBILE APPLICATION (GESMO)}

\subsection{Requirements}

We implemented GESMO using Android SDK 4.3, Eclipse IDE and testing done on MicroMax A106 smart phone. This application requires accelerometer and gyroscope sensors in mobile phone to run it. 


\subsection{Architecture \& Flowchart of GESMO}

Figure 1. shows the block diagram of GESMO application. The main stages of this app are detection and recognition of gesture made in the air, assign desired app to made gesture, compare and open the desired app. Initially, this detects and recognizes the gesture in which the user desires to make in the air, and then assign this gesture for any app. Next store that pattern and application in SQLite database for future comparison as shown in Table I consisting application name, package name and air gesture pattern. Then if user wants to open a particular app, he must gesture or draw that pattern in the air; at this moment system compares this pattern with predefined gesture from the SQLite database. When both the gestures match, open the desired application. We represented the above discussion using flowchart given in figure 2 .

Table 1. Patterns in Database

\begin{tabular}{|c|l|l|c|}
\hline Id & $\begin{array}{c}\text { Application } \\
\text { Name }\end{array}$ & $\begin{array}{c}\text { Package } \\
\text { Name }\end{array}$ & $\begin{array}{c}\text { Air } \\
\text { Gesture }\end{array}$ \\
\hline 1 & whatsapp & com.whatsapp & $\mathrm{w}$ \\
\hline 2 & MX player & $\begin{array}{l}\text { com.mxtech.ved } \\
\text { eoplayer.ad }\end{array}$ & $\mathrm{m}$ \\
\hline 3 & --- & --- & --- \\
\hline
\end{tabular}

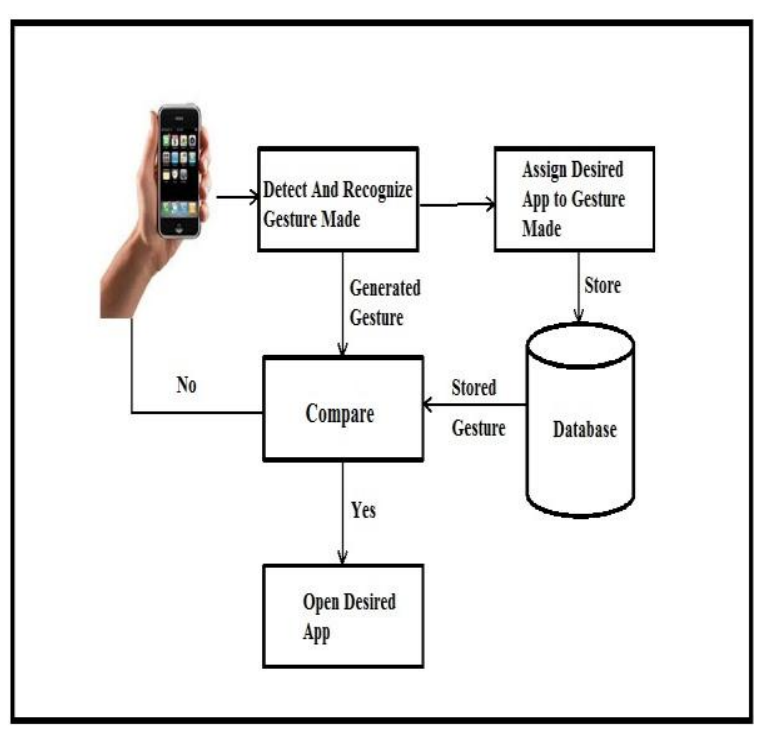

Fig. 1. Block Diagram of GESMO

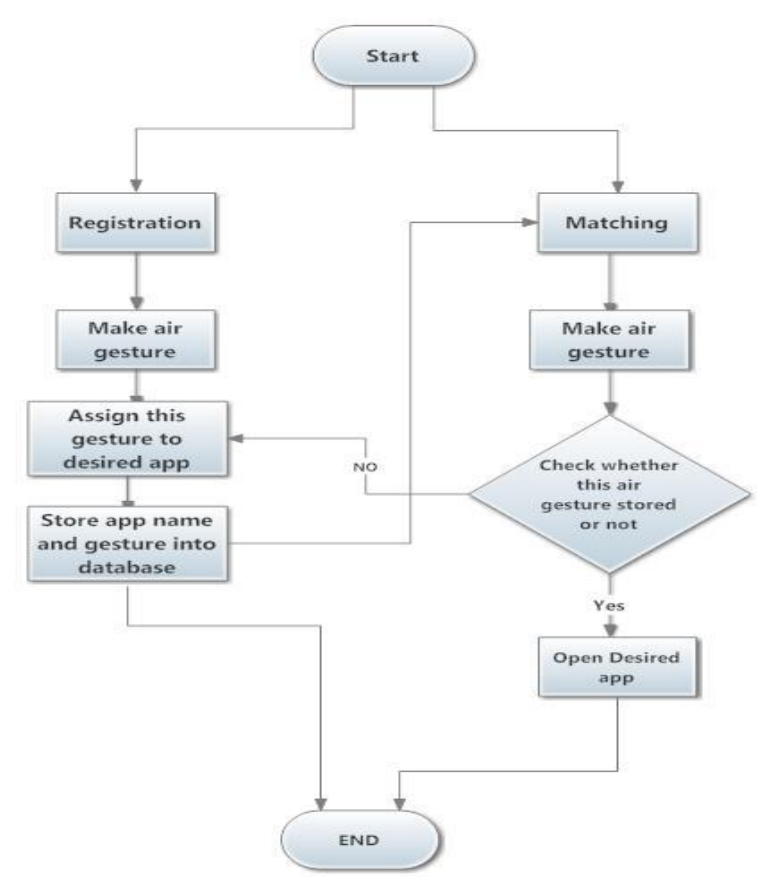

Fig. 2. Flowchart of GESMO

For GESMO, we make use of in-built accelerometer and gyroscope sensors from the mobile devices. Here an accelerometer and gyroscope sensors are hardware sensors. An accelerometer measures linear acceleration (eg. Shake) and gyroscope sensor measures angular acceleration (eg. tilt). Accelerometer sensor provides continuous data as we require this sensor data further. For classification we used the Support Vector Machine (SVM) algorithm. To collect sensor data for SVM we used the touch event. When user holds touch screen event till one releases their finger from the screen, all the continuous stream of sensor data are to be stored in arraylist. At the end of this, sensor data and application name is stored into database. To open the required app, we used Hidden Markov Model (HMM) algorithm in recognition phase [6]. When user creates gesture in air then this gesture pattern compares with all gesture pattern stored in the SQLite database. Suppose SQLite database contains 5 stored gestures and when user creates a gesture in air then this gesture is compared with the all 5 stored gestures and calculates the percentage of matching. If percentage of matching is greater than $70 \%$ then required application will be launched.

\section{RESULTS AND DISCUSSION 4.1 Accelerometer reading}

This section presents graph showing values generated by Accelerometer sensor in the mobile device. Figure 3 shows the graph of accelerometer sensor values. Accelerometer sensor contains the three dimensional axes i.e. $\mathrm{x}, \mathrm{y}, \mathrm{z}$. Using these axes we plot a graph in which red lines shows the $\mathrm{x}$ axis, green lines shows the $\mathrm{y}$ axis and blue lines shows the $\mathrm{z}$ axis.

$$
\begin{aligned}
& \text { x - Red } \\
& \text { y - Green } \\
& \text { z - Blue }
\end{aligned}
$$




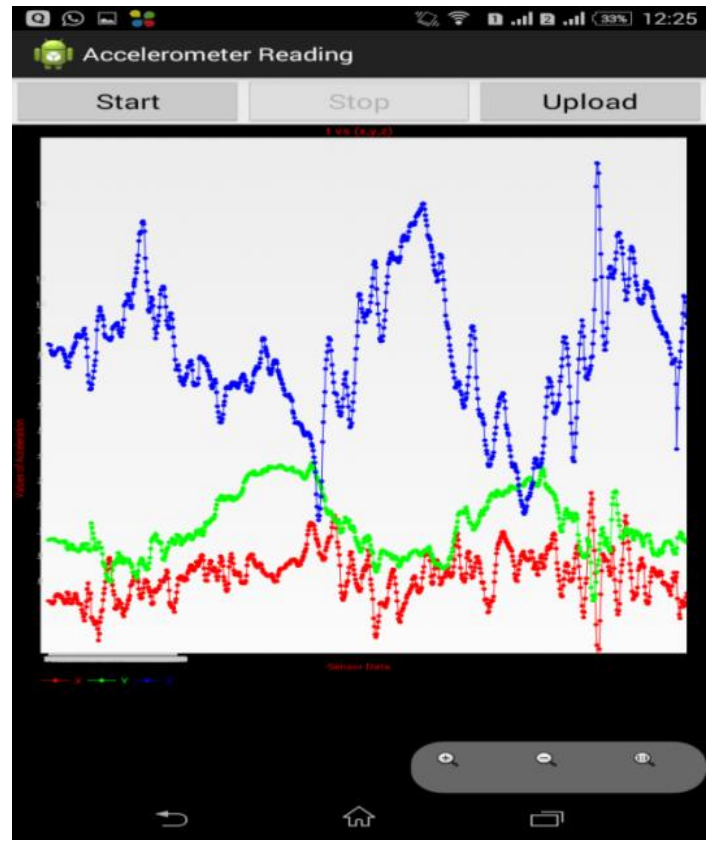

Fig. 3. Accelerometer Reading

\subsection{Snapshots of GESMO application}

Figure 4 i) shows the launcher icon of GESMO. After clicking this icon of app on app screen, home screen of GESMO is displayed to user. This GESMO icon indicates that GESMO app is successfully installed in smart phone. This icon gives shortcut to user to launch this application.

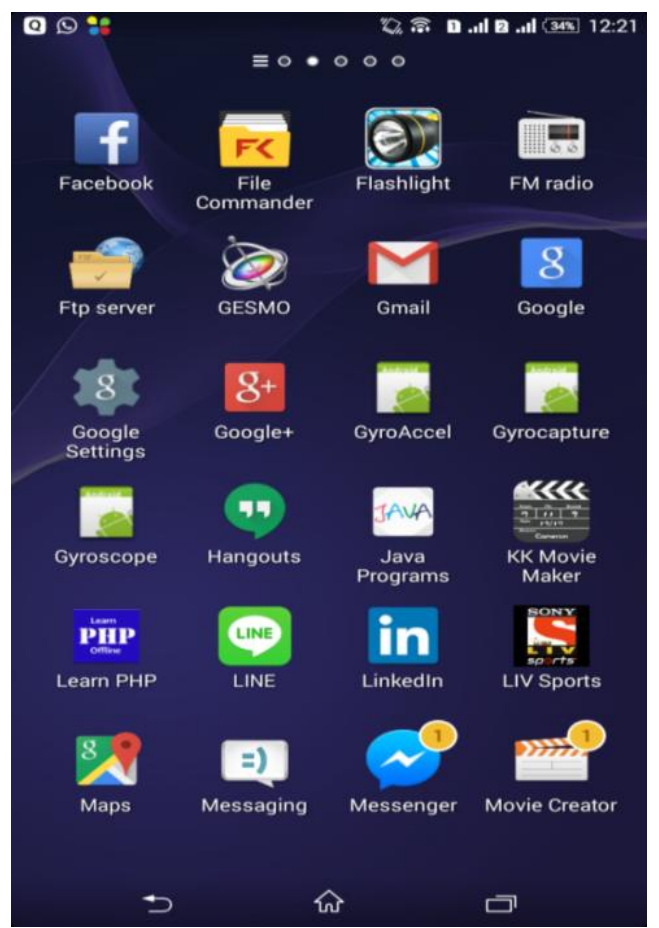

GESMO launcher activity

Figure 4 ii) shows the home screen of GESMO android application. At the top of this screen, values of $x, y, z$ axes of an accelerometer are displayed. Below these values we provided one list view that shows the air gesture stored for the said application. The list view displays name of android application and inside the square bracket we display initial letter of application. At the bottom of home screen, it provides

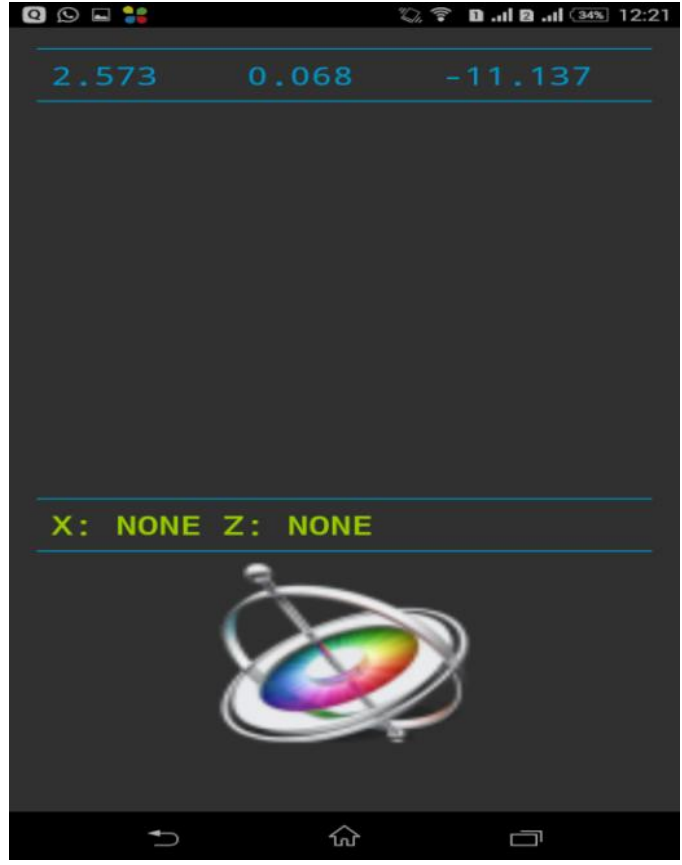

ii) Home Screen

the touch event so that user can touch on this screen to make gesture in the air. When user releases the finger from screen air gesture it will be stored into the database.

Figure 4 iii) shows the single screen i.e. Activity. This Screen displays the list of all android applications pre-installed in smart phone. This screen appears after the above screen and

here, user selects one particular application from the list. The name of the application and air gesture is stored into the database.

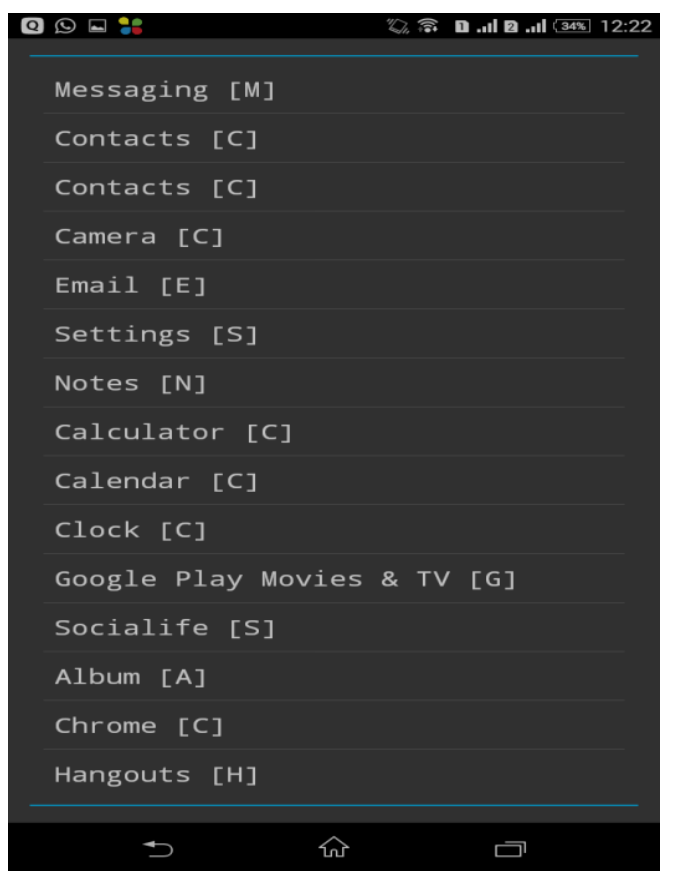

\section{iii) List of installed App}

Figure 4 iv) shows the current status of GESMO indicating that the air gesture $\mathrm{M}$ is stored for launching Messaging application similarly air gesture $\mathrm{W}$ is stored for launching WhatsApp application. If user wants to delete this 
applications from GESMO database then this name of application and air gesture pattern is deleted by simply clicking on this list view. Figure 4 v) and vi) shows Messaging application and WhatsApp are successfully launched by using GESMO application.

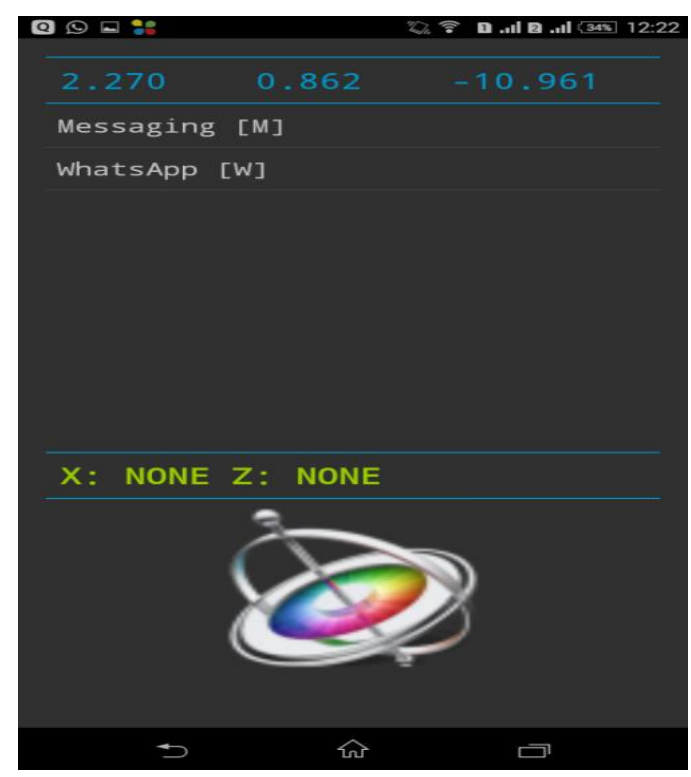

iv) Home Screen with Defined Gesture

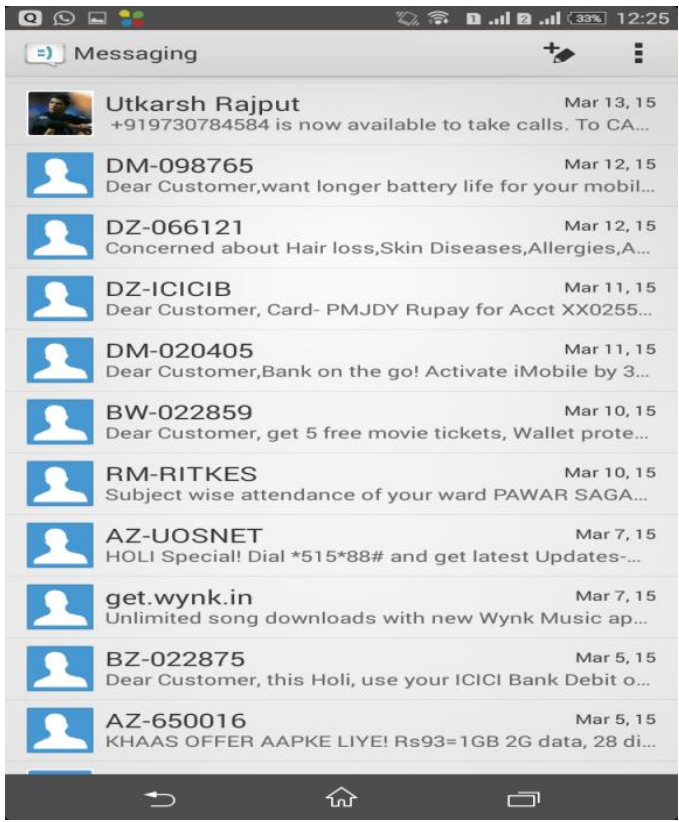

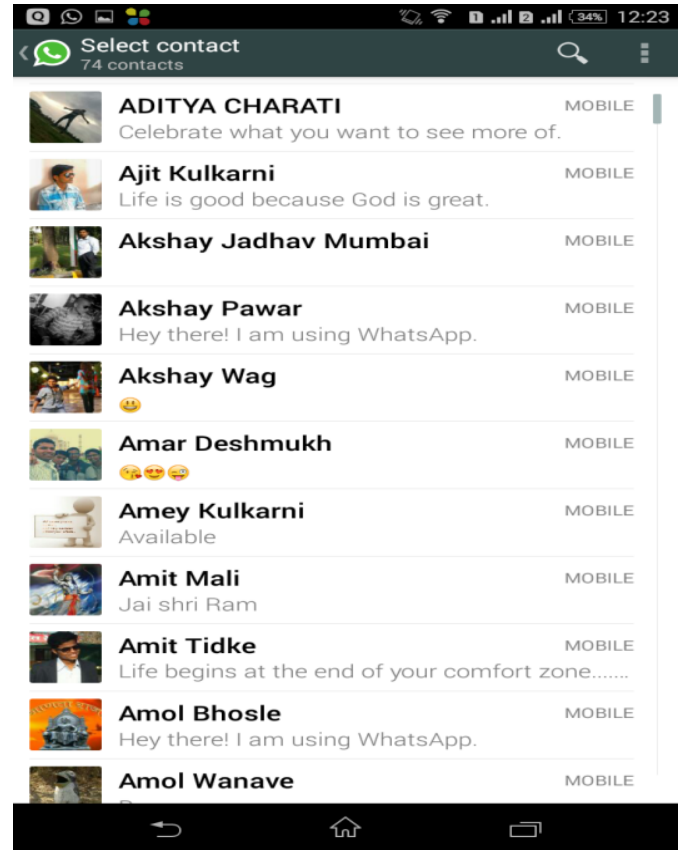

$\mathrm{v}$ and vi) Launched Applications

Fig. 4 Snapshots of GESMO app

\section{ADVANTAGES AND DRAWBACKS OF GESMO}

- Helpful for blind people.

- It feels more natural and cool app.

- New Technology for launching android application.

- User can store single stroke English letter as an air gesture for launching android application as well as user can store alphabets in Marathi and shapes e.g. Triangle, square.

- Useful in emergency without display screen.

To run GESMO, there must be accelerometer, gyroscope sensor embedded in your Smartphone. There is scope to improve this input technology by using other type of sensors.

\section{CONCLUSION AND FUTURE WORK}

The paper presents a mobile application which attempts to exploit the inbuilt mobile sensors accelerometer and gyroscope to detect one stroke gestures made in air with the help of mobile. In order to identify start and end point of motion, we provide press and hold mechanism. Hence user is able to draw one stroke letter in an air to open a corresponding app. Here the recognition rate of most of the gestures is between 70-80 \% which is an acceptable range. Also, we have given flexibility to user that in which they can decide letter for his app. This is useful when there are more than one applications starting with same letter. In near future we will be aiming to recognize multi stroke letters and words which will be more complex. Also, for authorization we are planning to support signature gestures to get the secure access of mobile device.

\section{ACKNOWLEDGMENTS}

We would like to thank Mr.Vishwanath Awate, founder of Agile Technologies, Pune for his guidance and support. Also, we render our gratitude to Department of CSE and IT, 
Rajarambapu Institute of Technology, Rajaramnagar, Maharashtra, India.

\section{REFERENCES}

[1] Bin Peng; JinmingYue; Chen Tianzhou, "The Android Application Development College Challenge," High Performance Computing and Communication \& 2012 IEEE 9th International Conference on Embedded Software and Systems (HPCC-ICESS), 2012 IEEE 14th International Conference on , vol., no., pp.1677,1681, 25-27 June 2012

[2] Saxena, A.; Jain, D.K.; Singhal, A., "Hand Gesture Recognition Using an Android Device," Communication Systems and Network Technologies (CSNT), 2014 Fourth International Conference on , vol., no., pp.819,822, 7-9 April 2014

[3] Jiahui Wu; Gang Pan; Daqing Zhang; Guande Qi; Shijian $\mathrm{Li}$, "Gesture Recognition with a 3-D Accelerometer", Ubiquitous Intelligence and Computing 6th International Conference on, pp 25-38, 2009.
[4] Sandip Agrawal; Ionut constandache; Shravan Gaonkar; Romitroy Choudhuri; Kevin Caves; Frank DeRuyter, "Using Mobile Phones to Write in Air", MobiSys's 11, Proceedings of the 9th international conference on Mobile systems, applications, and services, ACM June 28- July 12011.

[5] Po-Kuan Huang; Tung-Yang Lin; Hsu-Ting Lin; ChiHao Wu; Ching-Chun Hsiao; Chao-Kang Liao; Lemmens, P., "Real-time stereo matching for 3D hand gesture recognition," SoC Design Conference (ISOCC), 2012 International, vol., no., pp.29,32, 4-7 Nov. 2012.

[6] Amma, C.; Georgi, M.; Schultz, T., "Airwriting: HandsFree Mobile Text Input by Spotting and Continuous Recognition of 3d-Space Handwriting with Inertial Sensors," Wearable Computers (ISWC), 2012 16th International Symposium on, pp.52,59, 18-22 June 2012.

[7] Meenaakumari. M; M. Muthulakshmi, "MEMS ACCELEROMETER BASED HAND GESTURE RECOGNITION", International Journal of AdvancedResearch in Computer Engineering \& Technology (IJARCET), Volume 2, No 5, May 2013. 